THE AsTrophysical JouRnaL, 499:728-730, 1998 June 1

(C) 1998. The American Astronomical Society. All rights reserved. Printed in U.S.A.

\title{
LARGE MAGELLANIC CLOUD MICROLENSES: DARK OR LUMINOUS?
}

\author{
ANDREW GOULD ${ }^{1}$ \\ Department of Astronomy, Ohio State University, 174 West 18th Avenue, Columbus, OH 43210; gould@astronomy.ohio-state.edu \\ Received 1997 October 2; accepted 1997 December 30
}

\begin{abstract}
Zhao has proposed that the microlensing events observed toward the Large Magellanic Cloud (LMC) could be due to faint stars in a dwarf galaxy or tidal debris lying along the line of sight to the LMC. Zaritsky \& Lin claim to have detected such a structure which, they believe, could account for most of the observed microlensing optical depth. Here I show that a large-area surface-brightness map made by de Vaucouleurs constrains any such structure to one of four possibilities. Either (1) it does not account for a significant fraction of the observed microlensing, (2) it covers the inner $\sim 3^{\circ}$ of the LMC but does not extend beyond $\sim 5^{\circ}$ from the LMC center, (3) it is smooth on scales of $\sim 15^{\circ}$ in both transverse directions, or (4) it has a stellar mass-to-light ratio which exceeds by a factor $\gtrsim 10$ that of known stellar populations. The second and third possibilities would not be expected to apply to tidal debris. The last merely rephrases the dark-matter problem in a new form.
\end{abstract}

Subject headings: dark matter - Galaxy: halo — gravitational lensing — Magellanic Clouds

\section{INTRODUCTION}

The MACHO (Alcock et al. 1997a) and EROS (Aubourg et al. 1993) collaborations have detected a total of 16 candidate microlensing events toward the Large Magellanic Cloud (LMC). While a few of these may be variable stars, the great majority are likely to be genuine microlensing. MACHO has estimated an optical depth of $\tau=2.9_{-0.9}^{+1.4}$ $\times 10^{-7}$ based on a subset of these detections, a significant fraction of the $\tau \sim 4.7 \times 10^{-7}$ expected if the dark halo of the Milky Way were composed entirely of massive compact halo objects (MACHOs). Sahu (1994) and Wu (1994) suggested that a large fraction of the events could be due to lensing by stars within the LMC itself, particularly in the LMC bar, and indeed one event appears to be due to a binary in the LMC (Alcock et al. 1997a). However, general dynamical arguments constrain the self-lensing of the LMC disk to $\tau_{\text {self }} \lesssim 1 \times 10^{-8}$ (Gould 1995). Moreover, as observations have continued it is becoming apparent that the events do not occur preferentially in the bar which is what one would expect if they were due primarily to LMC stars. Some events are also expected from stars in the Milky Way disk. However, based on star counts, Gould, Bahcall, \& Flynn (1997) estimate $\tau_{\mathrm{MW}} \sim 8 \times 10^{-9}$. This is about 35 times smaller than the observed value, although Gould et al. (1997) argue that one of the observed events may well be due to a disk $M$ dwarf. In brief, the majority of these events do not seem to be due to known stellar populations. This is an important and puzzling result because the estimate of the typical mass of the lenses (derived from the observed timescales of the events and dynamical models of the Galactic halo) is $\sim 0.4 M_{\odot}$. If these objects were composed of hydrogen they would burn, and the population would easily be detected (assuming it were distributed throughout the Galaxy). If they are some new exotic object, it is most curious that they have the mass of normal stars.

This puzzle led Zhao (1997) to suggest that the events may be due to a dwarf galaxy or tidal debris from a disrupted galaxy along the line of sight to the LMC. The existence of the Magellanic Stream (the full extent of which is

\footnotetext{
${ }^{1}$ Alfred P. Sloan Foundation Fellow.
}

$\sim 10^{\circ} \times 100^{\circ}$ ) demonstrates the presence of substantial tidal debris from the Magellanic Clouds themselves. Although to date no stars have been detected in the Magellanic Stream, it could in principle have a stellar component which would likewise be extended. The debris could also be from an unrelated galaxy which, like the Sagittarius dwarf $\left(\sim 5^{\circ} \times 20^{\circ}\right)$, might be substantially extended along its orbit. Such structures would appear to explain in a natural way why the inferred masses are similar to those of stars: the lenses are stars. More recently, Zaritsky \& Lin (1997) claim to have detected such a foreground structure (but see Alcock et al. 1997b and Beaulieu \& Sackett 1997). They observed a field $\sim 2^{\circ}$ northwest of the LMC bar and found that $\sim 5 \%$ of the clump giants are brighter than the mean by 0.9 mag. They interpret this as evidence for a stellar population $0.9 \mathrm{mag}$ in the foreground. They estimate a stellar surface mass density of $\Sigma \sim 16 M_{\odot} \mathrm{pc}^{-2}$ which would account for a large fraction of the microlensing events. This conclusion is very appealing in that it would eliminate the need for exotic objects.

However, the problem of the "dark matter" apparently being detected in microlensing experiments is not diminished by positing that this mass lies in previously unrecognized structures. To be a natural solution to the problem, these structures must also have normal mass-to-light ratios. If newly found structures such as the one claimed by Zaritsky \& Lin (1997) were in fact responsible for the microlensing events, but they had anomalously high mass-to-light ratios, the "dark matter" mystery would simply take on a new form. I therefore investigate what limits can be placed using existing data on the mass-to-light ratio of previously unrecognized structures.

\section{MASS AND LIGHT}

If a structure, particularly tidal debris, lay 10 or $20 \mathrm{kpc}$ in front of the LMC, one would not expect its angular extent to be perfectly coincident with that of the LMC. In general it should, like the Magellanic Stream, extend well beyond the LMC. The surface brightness of any structure extending beyond $\sim 5^{\circ}$ from the LMC center is constrained by the LMC surface-brightness map of de Vaucouleurs (1957) to be fainter than $R \gtrsim 25 \mathrm{mag} \operatorname{arcsec}^{-2}$, the last isophote of 
the map. Assuming $V-R \sim 0.5$, this corresponds to $V \gtrsim$ $25.5 \mathrm{mag} \mathrm{arcsec}^{-2}$, or a surface brightness of

$$
S_{\max } \sim 2.2 L_{\odot} \mathrm{pc}^{-2},
$$

where I have used the identity

$$
V=26.4 \mathrm{mag} \operatorname{arcsec}^{-2}-2.5 \log \frac{S}{L_{\odot} \mathrm{pc}^{-2}} .
$$

On the other hand, to account for a fraction $f$ of the observed microlensing optical depth $\tau=2.9 \times 10^{-7}$ by a stellar structure at a distance $d_{\mathrm{ol}}$ requires a surface density $\Sigma$ (Paczyński 1986)

$$
\Sigma=\frac{f \tau c^{2}}{4 \pi G D}=47 f\left(\frac{D}{10 \mathrm{kpc}}\right)^{-1} M_{\odot} \mathrm{pc}^{-2},
$$

where $D \equiv d_{\mathrm{ol}} d_{1 \mathrm{~s}} / d_{\mathrm{os}}$ and $d_{\mathrm{ol}}, d_{\mathrm{ls}}$, and $d_{\mathrm{os}}$ are the distances between the observer, the lensing structure, and the LMC sources. Hence, the stellar mass-to-light ratio of the structure is bounded by

$$
\frac{M}{L}>22 f\left(\frac{M}{L}\right)_{\odot}=12 f\left(\frac{M}{L}\right)_{\mathrm{Mw}},
$$

where I have normalized the mass-to-light ratio to that observed for stars in the disk of the Milky Way. This is obtained by dividing the observed stellar column density of the local disk $\Sigma_{\mathrm{MW}}=27 M_{\odot} \mathrm{pc}^{-2}$ (Gould, Bahcall, \& Flynn $1996,1997)$ by the observed surface brightness of the local disk, $S_{\mathrm{MW}}=15 L_{\odot} \mathrm{pc}^{-2}$ (Binney \& Tremaine 1987), that is $(M / L)_{\mathrm{MW}}=\Sigma_{\mathrm{MW}} / S_{\mathrm{MW}}=1.8(M / L)_{\odot}$.

The primary evidence for the Zaritsky \& Lin (1997) structure is an apparent population of red clump stars, so the appropriate normalization in equation (2.4) is the $M / L$ of the Milky Way disk, the only Population I structure whose stellar content has been measured. More generally, one might consider more metal-poor structures such as disrupted dwarf spheroidals. Metal-poor systems generally have a higher ratio of low-mass to high-mass stars (Gould, Flynn, \& Bahcall 1998; Chabrier \& Méra 1997) and, of course, no recent star formation. Both characteristics lead to a higher stellar mass-to-light ratio. However, C. Pryor's (1996 private communication) preliminary estimate of the dynamical-mass-to-light ratio of typical globular clusters is $M / L \sim 2.5-3$. One might argue that globular clusters are depleted of low-mass stars relative to dwarf spheroidals by evaporation. However, most of the measured mass functions of globulars are unbroken power laws (Chabrier \& Méra 1997) indicating that evaporation has generally not been severe. In brief, there is no hard evidence for a stellar $M / L>3$.

To avoid clutter, I have not in this section propagated the errors in MACHO's optical depth estimate $\tau=2.9_{-0.9}^{+1.4}$ $\times 10^{-7}$. However, I remind the reader that this estimate is based on only a handful of events and could be revised substantially in the future.

\section{DISCUSSION}

Equation (2.4) implies one of four conclusions. Either:

1. The structures along the line of sight toward the LMC have a normal stellar mass-to-light ratio but in total account for only a fraction $f \lesssim 1 / 12$ of the observed microlensing events.
2. The argument of $\S 2$ fails because the intervening structures happen to be contained within $5^{\circ}$ of the LMC center (although they must extend over at least the inner $\sim 3^{\circ}$ to account for the observed events). This possibility could only apply to a self-gravitating structure and not to an intrinsically extended one such as tidal debris.

3. The argument of $\S 2$ fails because the intervening material is smooth on scales of $\sim 15^{\circ}$ in both directions, the size of de Vaucouleurs' (1957) map. Before constructing the LMC isophotes, de Vaucouleurs had to remove a smooth foreground component which has a mean surface brightness of $R \sim 21.2 \mathrm{mag} \operatorname{arcsec}^{-2}$ with a gradient of $\sim 15 \%$ across the field. The mean surface brightness is primarily due to the sky, and de Vaucouleurs (1957) assumed that the gradient is due to Galactic foreground. (The direction of the gradient is toward the Galactic plane.) However, if there were any other foreground structures that were smooth on the scales of the map, these would have been removed also. For example, Evans et al. (1997) have proposed that a warped and flared Milky Way disk is responsible for the LMC microlensing events. Such a structure would not be detected on the de Vaucouleurs (1957) map because, like the conventional disk, it has only a gentle gradient. On the other hand, while most tidal-debris structures like Magellanic Stream stars or a disrupted or quasi-disrupted galaxy (such as the Sagittarius dwarf) could well be smooth on scales of $15^{\circ}$ along the direction of their orbits, they would be relatively compact in the orthogonal direction. In fact, a structure with the surface-density profile of either of the Magellanic Stream or the Sagittarius dwarf would easily be detected in the de Vaucouleurs (1957) map.

4. There are tidal structures along the line of sight as suggested by Zhao (1997) and Zaritsky \& Lin (1997), but they have a mass-to-light ratio one order of magnitude higher than the local disk. They could then account for the observed microlensing events, but would be composed primarily of compact dark objects.

How do these conclusions square with the claims of Zaritsky \& Lin (1997) to have detected a structure with $\Sigma=16 M_{\odot} \mathrm{pc}^{-2}$ ? First note that at the center of the field, the LMC has a surface brightness $R \sim 22.4$ mag $\operatorname{arcsec}^{-2}$ (de Vaucouleurs 1957). The foreground structure has $\gtrsim 20$ times fewer stars, but these are 0.9 mag brighter, implying a surface brightness $R \sim 24.7 \mathrm{mag} \operatorname{arcsec}^{-2}$ which is close to the limit derived in $\S 2$.

There are, however, several problems with the Zaritsky \& Lin (1997) mass estimate of the LMC surface mass density, which taken at face value implies $M / L=6(M / L)_{\odot}$ for both the LMC disk and, by extension, the foreground structure. First, in deprojecting the LMC disk (assumed to be inclined at $i \sim 33^{\circ}$ ) Zaritsky \& Lin (1997) used csc $i$ rather than sec $i$. If one carries through their calculation but makes this one correction, one finds an LMC density of $\Sigma=103 M_{\odot} \mathrm{pc}^{-2}$ rather than 159. This still implies a stellar mass-to-light ratio $M / L \sim 4(M / L)_{\odot}$ which is about twice that of the Milky Way disk. Second, they made their calculation assuming that all the dynamical mass is in stars. That is, they assumed that the LMC does not contain any dark matter or gas at the location of their field. This assumption is sometimes regarded as plausible for the inner parts of galaxies and, as noted above, would imply a stellar mass-tolight ratio only about twice that of the Milky Way disk. However, given the flat LMC rotation curve, it also implies 
that the surface mass density falls inversely with radius. If, in fact, there were no dark matter, this would mean that the surface brightness should fall at the same rate (assuming a constant mass-to-light ratio). Actually according to the map of de Vaucouleurs (1957), it falls much faster than this which indicates substantial quantities of dark matter. Thus, $M / L<4(M / L)_{\odot}$ and there is therefore no evidence that the mass-to-light ratio of the LMC stellar disk differs substantially from that of the Milky Way. Finally, even if the stellar mass-to-light ratio were 6 as Zaritsky \& Lin (1997) derived, this would explain less than half of the optical depth. In sum, the apparent tenfold discrepancy between the estimates of Zaritsky \& Lin (1997) and this paper proves to be a combination of several factor $\sim 2$ effects.

I would like to thank Nathalie Palanque-Delabrouille who drew my attention to the work of de Vaucouleurs and Piotr Popowski and Gerald Newsom for careful readings of the manuscript. This work was supported in part by grant AST 94-20746 from the NSF and in part by grant NAG 5-3111 from NASA.
Alcock, C., et al. 1997a, ApJ, 486, 697 1997b, ApJ, 490, L59

Aubourg, E., et al. 1993, Nature, 365, 623

Beaulieu, J.-P., \& Sackett, P. D. 1997, AJ, submitted (astro-ph 9710156)

Binney, J., \& Tremaine, S. 1987, Galactic Dynamics (Princeton: Princeton Univ. Press)

Chabrier, G., \& Méra, D. 1997, A\&A, 328, 83

de Vaucouleurs, G. 1957, AJ, 62, 69

Evans, N. W., Gyuk, G., Turner, M. S., \& Binney, J. J. 1997, Nature, submitted

\section{REFERENCES}

Gould, A. 1995, ApJ, 441, 77

Gould, A., Bahcall, J. N., \& Flynn, C. 1996, ApJ, 465, 759 1997, ApJ, 482, 913

Gould, A., Flynn, C., \& Bahcall, J. N. 1998, ApJ, submitted (astro-ph 9711263)

Paczyński, B. 1986, ApJ, 304, 1

Sahu, K. C. 1994, Nature, 370, 275

Wu, X.-P. 1994, ApJ, 435, 66

Zaritsky, D., \& Lin, D. N. C. 1997, AJ, 114, 2545

Zhao, H. 1997, MNRAS, submitted (astro-ph 9703097) 